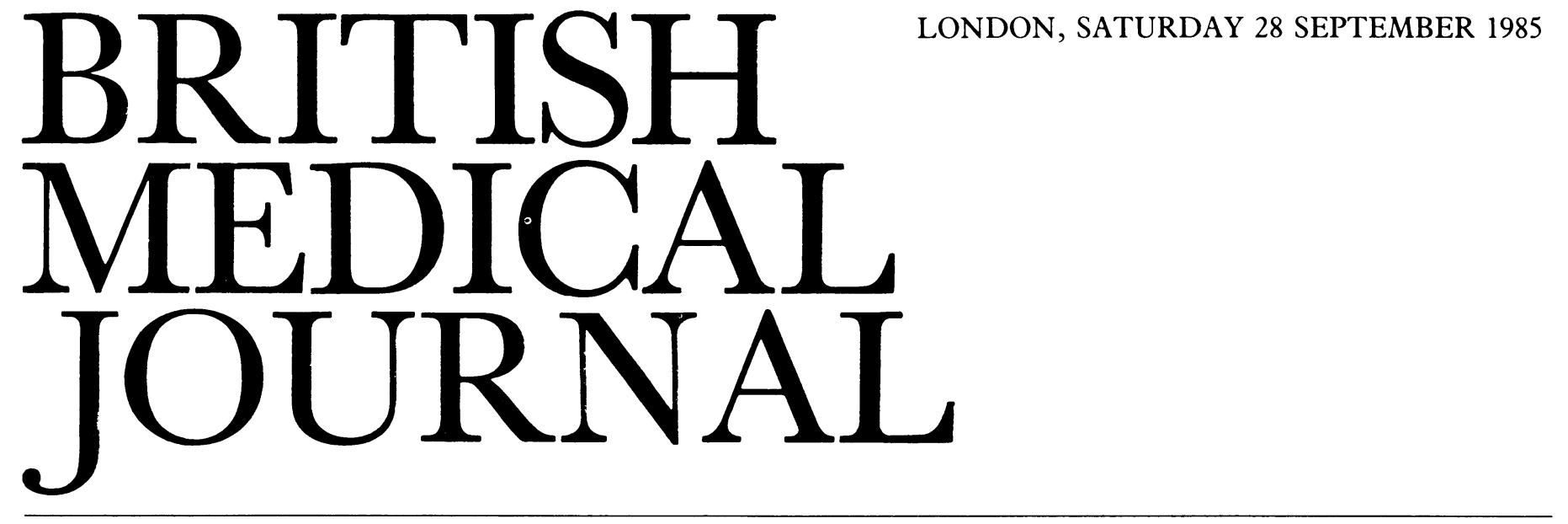

\title{
Red for danger: blood transfusion and colorectal cancer
}

The ability to differentiate the value of a clear set of observations from the inferences which may be drawn from them is the hallmark of a physician with "good clinical judgment." This type of discernment is also needed for retrospective observational clinical studies. Prospective controlled trials should investigate a hypothesis which has some a priori reason to support it, but those concerned with retrospective analyses have to be satisfied with generating new ideas to be tested. A good recent example of this process comes from several studies which have shown that patients with colorectal cancer survive longer if they are not given blood tranfusions at the time of their surgery. ${ }^{1-3}$

The research workers have not only suggested that this finding may be causally related but also considered the possibility that their observation might be an epiphenomenon: it might be that in each histopathological stage those patients needing blood transfusions had an intrinsically worse prognosis than those patients in the same stage who did not need them. Possibly a cumulative effect in the population as a whole has simply identified those patients with an inherently poorer outlook. The size of the difference in prognosis suggests, however, that this observation is not just a statistical statement of minor clinical importance but may be one of the crucial factors determining outcome, with an up to fourfold effect on tumour recurrence between the two groups $^{1}$ and a large influence on survival. ${ }^{2}$ Furthermore, their conclusion that perioperative blood transfusion has an independent influence on prognosis is supported by the statistical methods used-namely, the log rank and Cox proportional hazard regression analyses, which allow the more usual single variate analysis to be followed by multivariate adjustments made for all significant covariance simultaneously. ${ }^{4}$

Yet if it is assumed that the differences observed were directly related to blood transfusion the biological basis for this outcome may be difficult to explain. A simple analogy to the beneficial effects of blood transfusion in asscciation with kidney transplantation is attractive. That would suggest that the transfused blood (probably the white blood cells) results in immunosuppression, releasing the tumour to metastasise and grow.

Several different types of immunological events have been described after blood transfusion in patients given renal transplants. ${ }^{5}$ One possibility is that the beneficial effects of blood transfusion on the survival of kidney grafts may be attributed to clonal deletion and not to immunosuppression. ${ }^{6}$ Terasaki has argued that "multiple transfusions are postulated to hyperimmunise patients. Upon transplantation, a vigorous anamnestic response occurs. High dose immunosuppression given at transplantation kills or inactivates clones of reactive cells. In non-transfused patients, the peak immunosuppression at transplantation is given prematurely before rejection occurs." Others have suggested a process of patient selection which affects renal graft survival when blood transfusion is given before transplantation. ${ }^{7}$ After transfusion, and once broad spectrum HLA antibodies have developed, a patient will be difficult to match. Should a match be found, however, then the chances of graft survival will be enhanced. Setting the scene for these ideas some 40 years ago, Medawar clearly showed that blood transfusion is an immunostimulant in a "normal" host. ${ }^{8}$

At present, therefore, immunologists cannot readily explain either the effects of blood transfusion after renal transplantation or its deleterious effects on prognosis after resection for cure in patients with colorectal cancer. Possible answers to some of the questions might be obtained by a randomised controlled trial in which immunological tests could be carried out in parallel with randomly assigned clinical management.

For reasons argued elsewhere, however, ${ }^{9}$ and because of the well known "Hawthorne effect" associated with "open" studies, there seems little possibility of a truly controlled investigation of a patient population similar to that in the retrospective studies. The published data will persuade most surgeons with a responsible interest in colonic resection for cancer to correct preoperative anaemia or iron deficiency with haematinics, to redouble their efforts to prevent surgical bleeding, to resist the temptation to use blood transfusions of one or two units, and possibly to consider prior autoblood donation if preoperative anaemia is not a problem. ${ }^{1-3}$ To suggest that we should attempt randomised controlled trials for all these items before accepting their therapeutic value would be absurd. Possibly a trial of "washed" versus "unwashed" packed red cells might be justifiable. I suggest, 
however, that the real value of any such trials is that they encourage standardisation of management methods, including all the items mentioned above.

This conclusion might disappoint a trial technocrat, but it should be accepted as part of the difficulty and frustration inherent in clinical research. Furthermore, the scientific committees of grant giving bodies need to acknowledge more readily that, by its very nature, this type of clinical investigational work is not "clean" (in a scientific sense). Imaginative proposals should be encouraged to help generate reliable information based on some standardisation of treatment patterns, both operative and perioperative, which might help the outcome of the increasing numbers of patients requiring bowel resection. In any event, until it can be shown that blood transfusion is not harmful in the long term to patients with large bowel cancer, it seems reasonable to avoid it whenever possible.

L P FIELDING

Clinical Professor of Surgery at Yale,

St Mary's,

Waterbury,

Connecticut 06702

USA

1 Blumberg N, Agarwal MM, Chuang C. Relation between recurrence of cancer of the colon and blood transfusion. Br.Med F 1985;290:1037-9.

2 Foster RS, Costanza MC, Foster JC, Wanner MC, Foster CB. Adverse relation ship between blood transfusions and survival after colectomy for colon cancer. Cancer $1985 ; 55$ ship betwe

3 Burrows L, Tartter P. Effect of blood transfusions on colonic malignancy recurrence rate. Lancet 1982;ii:662.

4 Kalbfleisch JD, Prentice RL. The statistical analy'sis of failure time data. New York: John Wiley and Sons, 1980: 16-9, 70-81

Woodruff MFA, Van Rood JJ. Possible implications of the effect of blood transfusion on allograft survival. Lancet 1983;i:1201-2

6 Terasaki PI. The beneficial transfusion effect on kidney graft survival attributed to clonal deletion. Transplantation 1984;37:119-25.

Kirschenbaum AM, Schanzer H. Blood transfusions and kidney transplantation: review of controversies. Mount Sinai f Med 1983;50:393-7.

8 Medawar PB. Immunity to homologous graft skin: II. The relationship between the antigen between blood and skin. Br f Exp Pathol 1946;27:15.

9 Dudley HAF. Trials and tribulations for surgeons. Br 7 Surg 1985;72:255.

\section{Seventh age itch}

"Sans teeth, sans eyes, sans taste, sans everything." Shakespeare knew that blunting of the senses was one of the hallmarks of old age, but there is one exception-itch. All too often it appears or is exaggerated in the elderly.

Pruritus is synonymous with itch but when used as a diagnostic term is applied to patients whose itch is unaccompanied by any visible primary skin disease. Many such elderly patients have little to see on their skins, but excluding a primary skin disorder is not as easy as it might seem since scratching may have caused more than scratch marks. "Prurigo" is an ill defined term which describes the excoriated pink papules which are often seen on itchy skin and which seem to be due to scratching. ${ }^{2}$ Lichenification-thickened skin looking like Morocco leather-is also due to prolonged scratching and rubbing. Similarly, purpura, broken hairs, and pigmentation may be secondary to repeated trauma.

On the other side of the coin, even the use of a lens will not prevent trained doctors with intact sensory faculties from falling into some common traps. All of us can tell embarrassing stories about missing scabies in clean people, forgetting to ask about infested pets, overlooking underclothes crawling with lice, omitting to check for contact with fibreglass, ${ }^{3}$ disregarding minor eczema, and failing to appre- ciate the existence of conditions such as winter itch ${ }^{4}$ and $\underline{\underline{T}}$ aquagenic pruritus. ${ }^{5}$

Even when these have been considered, however, many elderly patients still have no recognisable skin disease $c$ responsible for their itching. Between $16 \%$ and $50 \% \widehat{\widehat{O}}$ of patients investigated for pruritus have an underlying systemic disease. ${ }^{6}$ Well established causes are obstructive $\mathbb{\infty}$ jaundice, chronic renal failure, pregnancy, thyroid disease, m lymphoma, carcinomatosis, iron deficiency anaemia, 을 intestinal parasites, and diabetes. ${ }^{7}$ Rarer causes include $\overrightarrow{\vec{F}}$ polycythaemia, ${ }^{8}$ haemochromatosis, ${ }^{9}$ brain tumours (especially those infiltrating the floor of the fourth ventricle),${ }^{10}$ and drugs such as cocaine, morphine, and chloroquine. ${ }^{11}$ It $\overline{\bar{\omega}}$ follows that if a cause for the pruritus is still not evident after a good history and examination of the skin, then a detailed physical examination and some screening tests are needed. Investigations should include testing the urine for sugar and protein, a complete blood count and sedimentation rate, blood urea and liver function tests, thyroid function tests, examination of the stools for occult blood and parasites, and a chest radiograph. ${ }^{12}$

If these give no answer xerosis or mild asteatotic eczema should be considered again but a hard core of patients remain in whom no cause can be found. We must either accept that with advancing age the incidence of idiopathic pruritus becomes higher or recognise the existence of an entity, "senile pruritus," attributable perhaps to age associated degenerative changes in peripheral nerve endings. ${ }^{13}$ If this diagnosis is made the patient should be kept under review as an underlying cause may show up later.

When a cause is found its treatment may cure the associated itch-though in chronic renal failure and hepatobiliary disease the management of pruritus may be far from satisfactory. In the absence of a demonstrable cause then the of patient should be treated symptomatically. Extremes of temperature and rough underclothing should be avoided. Adding an oil to the bath water will prevent the skin from drying out too much after bathing. Cooling applications include $0.5 \%$ menthol in aqueous cream. Crotamiton or a mildly potent topical steroid may also be useful. Systemic antihistamines, particularly trimeprazine and hydroxyzine, are worth trying; their effect is probably due to their sedative action. Unfortunately, these non-specific measures are often disappointing. Although for reasons of euphony Shakespeare could not have added "avec itch" to his list of changing sensory faculties, many patients and doctors know only too well that itch may be the final indignity of the seventh age of man.

Professor of Dermatology,

J A A HunTER

Royal Infirmary

Edinburgh EH39YW

1 Shakespeare W. As you like it. In: Craig WJ, ed. The complete works of William Shakespeare. London: Oxford University Press, 1955:227 (II. vii. 166.)

2 Savin JA. The management of pruritus. Br Med f 1973;iv:779-80.

3 Abel RR. Washing machine and fibreglass. Arch Dermatol 1966;93:78.

4 Cunliffe WJ. The skin and the nervous system. In: Rook A, Wilkinson DS, Ebling FJG, eds. Textbook of dermatology. 3rd ed. Oxford: Blackwell Scientific Publications, 1979:2000.

Greaves MW, Black AK, Eady RAJ, Coutts A. Aquagenic pruritus. Br Med f 1981;282:2007-9.

6 Kantor GR, Lookingbill DP. Generalized pruritus and systemic disease. $\mathcal{F}$ Am Acad Demat 1983;9:375-82.

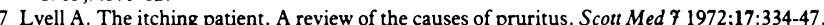

8 Fjellner B, Hagermark O. Pruritus in polycythaemia vera: treatment with aspirin and possibility of platelet involvement. Acta Derm Venereol (Stockh) 1979;59:505-12.

9 Nestler JE. Hemochromatosis and pruritus. Ann Intern Med 1983;98:1026.

10 Andreev VC, Petkov I. Skin manifestations associated with tumours of the brain. Br f Dermatol 1975;92:675-8

11 Bruinsma W. A guide to drug eruptions. Amsterdam: Excerpta Medica, 1973:76.

12 Champion RH. Generalised pruritus. Br Med $\mathcal{J}$ 1984;289:751-3.

13 Gilchrest BA. Pruritus. Pathogenesis, therapy and significance in systemic disease states. Arch Intern.Med 1982;142:101-5.

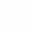

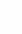

.

